Archives

9| 1992

Varia

\title{
Du bon usage du sacré : Érasme de Rotterdam
}

\section{André Godin}

\section{OpenEdition \\ Journals}

\section{Édition électronique}

URL : http://journals.openedition.org/ccrh/2803

DOI : $10.4000 /$ ccrh.2803

ISSN : $1760-7906$

\section{Éditeur}

Centre de recherches historiques - EHESS

\section{Édition imprimée}

Date de publication : 15 avril 1992

ISSN : 0990-9141

\section{Référence électronique}

André Godin, «Du bon usage du sacré : Érasme de Rotterdam », Les Cahiers du Centre de Recherches Historiques [En ligne], 9 | 1992, mis en ligne le 18 mars 2009, consulté le 20 avril 2019. URL : http:// journals.openedition.org/ccrh/2803; DOI : 10.4000/ccrh.2803

Ce document a été généré automatiquement le 20 avril 2019

Article L.111-1 du Code de la propriété intellectuelle. 


\title{
Du bon usage du sacré: Érasme de Rotterdam
}

\author{
André Godin
}

1 En guise d'introduction, quelques mots sur ce titre clin d'oeil et sur les raisons d'un choix.

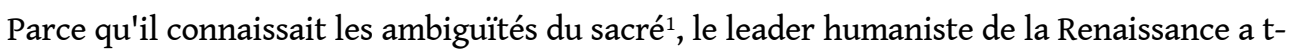
il seulement souhaité en voir tempérer l'emploi, comme Pascal voulait qu'on le fît des maladies ou le philosophe stoïcien des passions opposées à la raison?

2 En conformité avec le stéréotype confortable sur l'écrivain de Rotterdam frayant utopiquement et solitairement sa voie entre un bon et un mauvais usage de l'ensemble des expressions humaines d'un sacré encore prégnant dans l'Europe du XVI ${ }^{\mathrm{e}}$ siècle, faut-il se résoudre à ne voir en Erasme, traitant des cérémonies de l'Eglise catholique, que l'homme d'un juste milieu cotonneux, en somme ce chrétien réformiste, enfant terrible mais au fond très fidèle de l'Eglise, comme l'a campé en 1987 dans une biographie apologétique, Léon Halkin ${ }^{2}$, l'un des maîtres reconnus des études érasmiennes?

Mon propos n'est pas ici de rouvrir le débat largement dépassé et passablement étriqué sur le plus ou moins grand degré d'orthodoxie ou d'orthopraxie du catholicisme d'Erasme, à propos de sa critique de telle ou telle manifestation du sacré chrétien traditionnel. Peu ou prou, cela nous conduirait à préférer une notion fuyante à un véritable objet historique. Plutôt donc que d'en rester à des considérations générales sur la notion de sacré, véritable "entonnoir sémantique » pour reprendre la métaphore imagée de F. A. Isambert ${ }^{3}$, j'ai choisi d'aborder autrement la question du pèlerinage chez Erasme. Traiter du pèlerinage érasmien, ce pourrait être un bon medium de communication à propos d'une des actions rituelles les mieux attestées dans l'Orient et l'Occident d'hier et d'aujourd'hui. L'un des thèmes majeurs du grand historien méconnu Alphonse Dupront était précisément le pèlerinage et ses avatars historiques, en premier chef la croisade, cette figure paroxystique du pèlerinage ${ }^{4}$.

4 Pour situer le texte d'Erasme dont je propose ici l'analyse, rappelons qu'il est tiré des Colloques, au départ sorte de « Méthode Assimil » pour l'apprentissage du latin, élargie au fil des années en chronique littéraire de l'époque. Cette oeuvre de toute une vie 
renouvelle le genre du dialogue antique. A l'égal de L'Éloge de la folie, les Colloques furent un des best-sellers $d u \mathrm{XVI}^{\mathrm{e}}$ siècle et, en édition expurgée, ils restèrent à travers l'Europe d'Ancien Régime, l'un des instruments privilégiés de la pédagogie latine ${ }^{5}$.

5 Paru pour la première fois en 1526, le colloque "Pèlerinage pour cause de religion ", traite de quatre pèlerinages réputés : Saint-Jacques de-Compostelle, Mariastein près de Bâle, Notre-Dame de Walsingham et Saint Thomas de Cantorbéry. Erasme avait une connaissance directe de ces deux derniers sanctuaires qu'il visita vers 1512-1514, en compagnie d'amis anglais, parmi lesquels John Colet, pionnier de l'humanisme pédagogique en Grande-Bretagne ${ }^{6}$.

6 Au-delà des péripéties contingentes d'une lutte de $\operatorname{clercs}^{7}$, ce qui paraît durablement en question dans le pèlerinage de chrétienté, ne serait-ce pas la conception chrétienne du sacré et l'un des soubassements traditionnels d'une société de religion? D'où cette approche anthropologique pour repérer dans l'oeuvre, les traces d'un processus de désacralisation qui, avant et après Erasme, caractérise en profondeur l'histoire culturelle en Occident dans sa longue durée. C'est à l'intérieur de ce plus vaste horizon que je souhaite inscrire l'analyse de ce petit chef d'oeuvre érasmien.

7 Je propose donc une confrontation entre les données essentielles, quasiment immuables, du pèlerinage et le travail subtil d'escamotage, de dévalorisation mais aussi de transfert religieux, social, auquel se livre Erasme sur les lieux et objets sacrés.

8 Tout d'abord le titre: Peregrinatio religionis ergo, «Le pèlerinage pour cause de religion ». Pourquoi cette construction stylistique ${ }^{8}$ ? N'est-ce pas insinuer d'emblée qu'il existe des formes de pèlerinage qui n'ont pas pour but la religion, ce qu'Erasme appelle ailleurs le pèlerinage-prétexte ${ }^{9}$ ? Comme si la piété n'était pas le vrai motif! Avec Erasme le subtil, rien n'est indifférent, à commencer par le non-dit. Si peregrinatio est utilisé à six reprises, peregrinus n'apparaît nulle part. Curieuse, symptomatique, cette absence d'un mot caractéristique de la réalité et de l'imaginaire pèlerins ${ }^{10}$. On relève des termes de substitution ${ }^{11}$. S'ils n'accréditent pas absolument l'hypothèse d'un déficit sacral au niveau du langage, ces mots de substitution suggèrent quand même la question : par son refus d'employer le vocabulaire courant, Erasme n'est-il pas déjà en train d'occulter un fait de société ? En tout cas, au sens propre, littéral du terme, Erasme est anti-pèlerin.

L'attesterait encore la dénomination des deux locuteurs de la pièce : Ménédème, celui qui demeure dans son canton (le dème), le sédentaire qui se refuse à courir les chemins, véritable antitype du pèlerin; Ogyge, dont le nom grec évoque à la fois l'Ogygie, île homérique où règne Calypso et Ulysse le globe-trotter ${ }^{12}$. Ogyge est donc un personnage odysséen au comportement suranné. Ménédème-Ogyge: héros éponymes figurant le contraste entre d'une part l'idéal d'une société neuve, stable, raisonnable, très critique sur le mercantilisme pèlerin et, de l'autre, la fascination d'une société obsolète de l'extraordinaire poursuivi dans une errance sacrale incessante que récapitule Ogyge en sa fiévreuse déambulation à travers lieux et objets sacrés européens.

Innombrables sont les détails piquants, les traits furtifs ou les anecdotes déployées qui manifestent un observateur aigu de son siècle, survivances et décrochage par rapport à l'imaginaire collectif médiéval qu'incarnait puissamment le pèlerin chrétien. Décrochage, déchirure ou écart mais non rupture radicale avec le vieil ordre social de chrétienté. Témoins, ces élans de ferveur pèlerine qui soulèvent çà et là la pâte épaisse des faits et gestes du pèlerinage. On peut y découvrir, à juste titre, l'idéal érasmien d'un juste-milieu réformateur. Mais tout aussi légitimement, y repérer les tâtonnements d'un processus 
social plus ample qui, « comme tout ce qui touche au sacré, comporte des transferts ou des latences de resacralisation $»^{13}$.

11 Avec le premier pèlerinage évoqué, Saint-Jacques de Compostelle, le point de vue critique l'emporte. D'entrée de jeu, Ménédème raille l'accoutrement de son ami, enfin de retour, emberlificoté dans ses affutiaux de pèlerin: "Mais pourquoi cette parure? Te voilà couvert de coquilles creuses, tout garni d'images en étain et en plomb, décoré de colliers de paille ; au bras, il porte des oeufs de serpent » (11-13).

D'emblée donc, par son costume composite qui accumule de façon grotesque les signes de reconnaissance sociale du pèlerinage, l'homme-pèlerin se trouve dévalorisé. Ce déguisement bizarre déconcerte l'ami, signale une insane étrangeté qui déshumanise Ogyge, affublé de vils matériaux ${ }^{14}$. L'effet de dépersonnalisation semble d'ailleurs renforcé par la construction grammaticale qui hésite entre la troisième et la seconde personne des verbes employés. Le refus de la condition pèlerine, déjà prévisible par l'absence du mot "pèlerin ", est ici confirmé par la dérision de son signalement. De l'étrangeté dynamique enclose dans per-egrinus, Erasme ne retient que l'aspect matériel, visible, superficiel : il y a perte de sens. Au lieu d'être ontologique, la transformation s'inscrit dans le registre de l'apparence et d'une chosification cumulative qui singe le decorum humain, la bienséance sociale. Métamorphosé par ses horripaux de pacotille, Ogyge n'est plus pleinement sujet. Il ne tient même pas en mains le bourdon, cet autre insigne de l'homme qui parcourt l'espace sacral dont les innombrables chemins de SaintJacques ont inscrit la prégnance dans le paysage européen ${ }^{15}$. L'homo viator s'est changé en objet de curiosité et, pour un peu, en bête curieuse.

Débute alors, entre Ménédème et le pseudo-pèlerin, un véritable dialogue de sourds. A Ogyge qui énonce fièrement ses visites aux divers sanctuaires, Ménédème sussure ironiquement : « Pour te distraire, je suppose » - "Que non, réplique Ogyge, par piété » (17-18). Le cliquetis des formules latines symétriques, avec postposition comme dans le titre, met en valeur les deux mots du malentendu : animus et religio. Selon Ménédème, le pèlerinage relève de l'agrément personnel, voire du «tourisme spirituel ». Pour Ogyge, c'est un acte de religion désintéressé et solidaire. Il s'agissait pour lui, en effet, d'acquitter à Compostelle un vœu de sa belle-mère pour la réussite d'une naissance dans la famille, donc d'une forme de pèlerinage très courue au $\mathrm{xvI}^{\mathrm{e}}$ siècle: le pèlerinage votif par procuration. Ménédème amorce plaisamment sa critique en partant du topos des bellesmères abusives. Sous le cocasse de la situation apparaissent :

1 - la mise en cause de cette «nouvelle sorte de vœu » (32) qui entraîne la dépossession de soi ;

2-une certaine méconnaissance érasmienne de l'aspect communautaire voire " grégaire » de tout pèlerinage digne de ce nom. Les acteurs du pèlerinage érasmien sont Ogyge, flanqué de deux ou trois amis, et non les foules, à peine évoquées lors des deux premiers pèlerinages, physiquement absentes des deux derniers.

14 Cette scénographie anthropomorphe se développe en une interprétation naturaliste de la pingrerie du saint. A une question "piégée » sur la coquille creuse donnée par le saint préférentiellement à tout autre objet, Ogyge répond: «Parce qu'il en a des tas; la mer proche les apporte » (30). L'affabulation érasmienne signale ici allusivement le certificat de pèlerinage dont les chanoines de Saint-Jacques de Compostelle munissaient les « saints jacquaires " au terme de leur périple ${ }^{16}:$ substitution démythifiante qui pointerait, elle aussi, le décrochage humaniste d'avec cet autre trait commun du pèlerinage médiéval, le brevet qui attestait l'accomplissement du saint voyage et consacrait, non point tant un 
exploit individuel que la reconnaissance sociale d'un statut de l'exceptionnel dans une société de l'oral, magiquement respectueuse de la preuve écrite. Après la dérision du costume et l'escamotage du nom, voici l'effacement de la preuve institutionntlle : autant de traits qui éclairent la méconnaissance et le refus de la condition pèlerine.

Voici maintenant un autre signe de discrédit à implications plus concrètement sociales. Introduit par une nouvelle impertinence sur l'icône - saint Jacques ne va pas bien (44) - le propos débouche sur une évocation de la crise religieuse de la société chrétienne. A cause du luthéranisme, on vient moins à Santiago et si d'aventure on y salue encore le saint, on n'offre plus guère d'offrandes, "en répétant que cet argent est beaucoup mieux entre les mains des pauvres » (49). S'énonce là un critère d'utilité sociale ${ }^{17}$. Par rapport à notre hypothèse et quant à l'utilisation de l'argent pèlerin, le jugement de valeur ici esquissé signale un nouveau craquement culturel. Battant en brèche un rite séculaire du pèlerinage, une pratique collective nouvelle est enclenchée qui tend à séparer le geste orant du geste offrant, la prière et le don. L'objet sacré ne polarise plus vraiment, en tout cas plus uniquement, la pulsion collective.

16 Ménédème approfondit son travail de sape à propos d'un autre lieu de culte, Mariastein. Cette fois, la scénographie est centrée sur une lettre de plainte et de menaces, dictée à un ange par la Vierge de pierre et miraculeusement déposée sur la chaire d'un prédicateur, Glaucoplutus, en qui l'ensemble des commentateurs reconnait Zwingli, instigateur d'une réforme zurichoise marquée à ses débuts par des bavures iconoclastes ${ }^{18}$. Comme l'a souvent pointé une lecture théologienne, il est vrai que la lettre mariale, sous une forme paradoxale, trace un chemin réformiste entre le refus radical et les abus criants de la vieille dévotion chrétienne. Mais le cri d'âme à la fin de la lettre - «Tu ne m'expulseras pas sans chasser en même temps mon fils que je tiens dans mes bras » (124) - n'est-il pas quelque peu assourdi par la causticité générale du propos et le côté burlesque de toute la mise en scène ? C'est bien ce que pensèrent les censeurs parisiens d'Erasme, en pure perte d'ailleurs ${ }^{19}$. D'édition en édition, subsistait la dénonciation massive de tous les recours matériels, caractéristiques d'une religion populaire panique. La Vierge de pierre reçoit de partout les demandes les plus extravagantes, les plus impies, les plus scabreuses, car " parfois même, on demande à la Vierge des choses qu'un jeune homme pudique oserait à peine demander à une maquerelle » (86-87). Le catalogue des recours immoraux concerne les principaux corps de métier et brocarde les représentants de toutes les classes sociales. Il y a là, en creux, un appel érasmien à un culte marial plus désintéressé, une exigence de pureté réformatrice. Appel et exigence qui vont à l'encontre d'une saisie plus terre à terre du mythe chrétien par la démarche pèlerine vouée à l'assouvissement de ses besoins vitaux au-delà de la morale et de la bienséance religieuse.

Le récit des deux pèlerinages anglais va nous permettre d'affiner l'hypothèse de départ: sous le miroitement ludique du discours se laisse deviner un double travail: de désintégration du fonds sacral pagano-chrétien hérité du Moyen Age populaire et de mise à jour des valeurs spécifiques d'un christianisme épuré, spiritualisé, intériorisé. Du pèlerinage à Notre-Dame de Walsingham, je détacherai deux expressions contrastées : l'ostension des reliques, l'élan de la prière érasmienne. Chacune des visites aux nombreux reliquaires que compte ce grand sanctuaire s'accompagne de railleries amusées où s'inscrit le transfert du langage de l'irrationnel pèlerin à un discours d'ordre essentiellement humain. Voici donc nos pèlerins dans la chapelle aux prodiges. Le mystagogue fait vénérer la première relique : le majeur d'un doigt humain qu'il assure être celui de l'apôtre Pierre. Après une inspection attentive du doigt dont la dimension 
semblait gigantesque, Ogyge déclare : «Il a donc fallu que saint Pierre fût un homme d'une taille prodigieuse! A ce mot, l'un de nos compagnons partit d'un éclat de rire, ce dont je fus fort chagriné (250-252)». On mesurera mieux la portée de cette démystification si l'on se souvient que, dans la vie du pèlerinage, l'attouchement de l'objet sacral représente le temps fort de la rencontre surnaturelle, le moment exaltant de participation à la puissance divine enclose dans les restes du corps saint. Dans une profonde synthèse d'anthropologie religieuse, A. Dupront a noté, évoquant d'autres cas, que « nombre de ces reliques attestent qu'elles viennent de corps de géants : ce qui n'est point sans vertu sacrale, car le géant est le support naturel de la puissance physique et, comme l'a longtemps enseigné la mythique traditionnelle, il représente l'homme normal de l'humanité primitive, celle des commencements de l'exil édénique ${ }^{20}$.

Dans la conscience du pèlerinage érasmien, il y a refus et perte du contact physique avec les forces mystérieuses du supra-humain, perception toute naturelle de la chose sacrée. Celle-ci ne polarise plus un au-delà de la nature et même, lors d'une ostension ultérieure de "reliques crasseuses, fétides ou sanguinolentes", dit le texte $(650,763,818)$, l'objet sacré suscite franchement le dégoût. Perdue aussi la dramatique biologique qui animait la dévotion des pèlerins au précieux lait de la Vierge. Cette relique est l'objet d'une très longue présentation critique. La démythologisation s'effectue par petites touches ironiques qui enlèvent tout crédit au phénomène merveilleux. Première invraisemblance : la quantité. De même que les fragments de la vraie croix qui, réunis de tous les lieux où on les exhibe, empliraient à ras bord un cargo, le lait virginal s'est tellement multiplié que notre voyageur-pèlerin s'exclame : " $O$ mère si semblable à son fils, lui nous a laissé sur cette terre tellement de son sang ; une si grande quantité, il est à peine croyable qu'une seule femme n'ayant eu qu'un enfant ait pu la produire, même si le bébé n'avait rien bu!»(278-280). Deuxième invraisemblance: la qualité. Solide ou liquide, ce lait? Le dévoilement cocasse du miracle intervient au terme du récit circonstancié «joliment cohérent» déclare, pince-sans-rire Ménédème (399), des transmissions successives du lait inépuisable :

Og- On ajoutait cet argument non dépourvu d'éclatante piété : le lait de la Vierge que l'on montre en plusieurs autres endroits est certes digne de vénération, mais celui-ci bien davantage, car celui-là fut râclé sur des rochers tandis que celui-ci coula directement des seins de la Vierge.

Mé- Quelle preuve en avait-on?

Og- Oh ! C'est ce que racontait la nonne de Constantinople qui en avait fait don.

Mé- Et qui elle-même, sans doute, le tenait de saint Bernard.

Og- Je crois que oui.

Mé-Car étant adulte, il lui est arrivé de goûter le lait au même sein que têta l'enfant Jésus. Aussi m'étonnè-je qu'on le surnomme mielleux (mellifluus) plutôt que laiteux (lactifluus). Mais comment appeler lait de la Vierge ce qui ne s'est pas écoulé de ses mamelles?

Og- Il s'est effectivement écoulé ; mais recueilli par le rocher sur lequel, par hasard, la Vierge s'était assise pour allaiter, il se cailla, puis par la volonté de Dieu, il se multiplia (422-434).

$19 \mathrm{Au}$ beau milieu du désaveu historico-critique, Érasme a placé une prière, joyau de pure spiritualité ; en contraste avec les puérilités dévotieuses des gestes pèlerins, l'accent est mis sur le véritable esprit d'enfance (infantiam rationalem) : fervente supplique pour que les visiteurs, avides du lait de la doctrine évangélique ${ }^{21}$ progressent «vers l'Homme parfait, dans la force de l'âge, qui réalise la plénitude du Christ ${ }^{22}$. 
Voilà, certes, une allégorisation saisissante qui s'élance, à partir d'une réalité suspecte, vers la plus haute spiritualité. Mais le prix à payer pour cette métaphore paulinienne, c'est le quasi effacement de la Vierge lactante, le renoncement au lien biologique, charnel, avec la Mère, que recherchaient les foules du pèlerinage dans leur quête avide de forces vitales élémentaires. Les formes du sacré panique chères à la religion populaire sont ici subverties. Il en va de même lors de la remise à Ogyge d'une relique que le sousprieur tire de sa bourse : «Un morceau de bois prélevé sur une planche qui, à ce qu'il semble, avait servi de siège à la Vierge-mère. Une merveilleuse odeur prouvait illico que c'était une chose hautement sacrée.

Mé-Je peux le voir?

Og- Je veux bien, mais si tu n'es pas à jeun ou si, la nuit dernière tu as fait l'amour avec ta femme, je ne te conseillerais pas de le regarder.

Mé- Montre-le, il n'y a aucun risque. (480-487)»

21 Extraordinaire disqualification de l'acte pèlerin, car dans le «brut» du pèlerinage, quelles que soient les religions ou l'époque, il est courant d'emporter avec soi une particule prise dans le lieu sacré, pour conserver le fruit de la rencontre, une parcelle de la puissance sacrale, « désormais liée à la vie personnelle et neuve du pèlerin " ${ }^{23}$. En un propos graveleux, d'autant plus dévalorisant, Erasme rabat sur l'ordre naturel (un bois odoriférant) le monde du sacré et de ses mirabilia dont se saisissaient les foules pèlerines par une pulsion dévotionnelle pouvant aller jusqu'au rapt. Quoiqu'il en soit, le passage parut trop équivoque, l'irrévérence trop libertine aux yeux des éducateurs jésuites du XVII ${ }^{\mathrm{e}}$ siècle. Dans un de leurs recueils de Colloques expurgés "à l'usage de la jeunesse studieuse $~^{24}$, la «merveilleuse odeur » s'exhale non de la planchette sacrée mais « d'un fragment de rocher extrait du tombeau où fut déposé le corps très saint de Marie ». Cette substitution moralisatrice confirme à sa manière le travail paradoxal d'épuration auquel Erasme s'était livré sur les principales sacralités du pèlerinage. L'essentiel n'est sans doute pas la survivance, chez notre humaniste, de l'anticléricalisme gaillard des écrivains médiévaux, mais - par l'évocation incongrue de réalités corporelles reliées au sexe - la subversion du mystère biologique des sacralités dont cherchait à s'emparer la foule fervente des pèlerins.

De ces bouleversements d'un imaginaire sacral archaïque, témoigne encore la suite immédiate du dialogue introduisant une nouvelle séquence de désacralisation : la visite à la statue de Notre Dame vers laquelle se laisse conduire Ogyge: "Le sous-prieur me demanda si j'avais jamais vu les secrets de la Vierge. Cette expression me troubla; je n'osai pourtant lui demander ce qu'il entendait par «secreta Virginis». Car en des domaines tellement sacrés, une simple erreur de langage n'est pas exempte de péril. Je lui dis que je ne les avais pas vus, mais que j'étais extrêmement désireux de les voir " (493-497). Passage derechef assez scabreux ${ }^{25}$, remplacé par un inoffensif «sequere me $»^{26}$ dans l'édition expurgée des bons pères. Vient alors, dans notre séquence, au lieu de l'habituel catalogue de miracles dont sont truffés les récits médiévaux de pèlerinage, une longue discussion sur les miracles dont la Nature gratifie les humains, en l'occurence les pierres précieuses aux propriétés figuratives merveilleuses. Par rapport au monde des mirabilia divins où s'établissait de soi la vie du pèlerinage médiéval, s'opère ici un transfert significatif. Dans cette scénographie très développée, se laisse deviner une autre marque du procès de désacralisation en cours dans la modernité européenne du XVI siècle, car on peut y repérer, au choix, une tension entre l'ordre de la nature et celui de la surnature ou bien le discours complaisant d'une apologétique naturelle du miracle. Dans 
les deux cas, des effets de rationalisation semblent perceptibles. La question qui se pose ici - comme elle le reste sans doute à propos d'autres traits de l'humanisme chrétien - est la suivante : équilibre-t-il vraiment les suggestions naturalistes du sacré parsemées çà et là dans le colloque et la pure lecture symbolique chrétienne qu'il maintient au coeur de ces séquences pré-rationalistes?

La finale du pèlerinage à Walsingham est loin de permettre une réponse univoque. Je n'isolerai qu'un seul moment de cette ultime séquence : la visite du trésor marial, extrait de l'autel et offert aux yeux éblouis du pieux spectateur en un feu rutilant d'objets d'or, d'argent et de vermeil, soupesés, appréciés, identifiés un par un par le sous-prieur qui prend ici figure d'orfèvre, de collectionneur ou de guide de musée. C'en est assez pour qu'Ogyge trouve que son pèlerinage a été des plus heureux. Voyeur comblé, il a été repu de "spectacles » (574) et il emporte sa planchette de salut, ce don sans prix offert par la Vierge. Notons au passage, le contraste malicieux établi entre la modeste relique et les richesses dévoilées lors de ce pèlerinage-spectacle.

Mais, à propos du sanctuaire de Saint-Thomas qui dépasse en richesse celui de la Vierge de Walsingham, Erasme revient sur un point esquissé dans la présentation du pèlerinage de Compostelle, en précisant sa critique des effets socio-économiques pervers du pèlerinage. Le remède qu'il préconise peut nous sembler bien timide. Il n'en constitue pas moins un nouvel indice de décrochement laïcisant par rapport au fonctionnement sacral de l'institution pèlerine ou, en d'autres termes, l'amorce d'un transfert social de la sphère du religieux à l'ordre du profane. Au cours de la visite guidée du trésor de la cathédrale, Gratien, alias John Colet, scandalisé par l'étalage de tant d'or et d'argent accumulé, suggère au mystagogue de service un plan modéré d'assistance aux pauvres : prélèvement par don gratuit ou prêts remboursables sur une partie de la richesse somptuaire gelée dans le sanctuaire de Cantorbéry. Ménédème on s'en doute, est d'accord avec cette esquisse d'un programme réformiste de redistribution de la propriété ecclésiastique ${ }^{27}$. Quant à Ogyge, sa ferveur pèlerine est quelque peu écornée par un tel luxe ostentatoire. $\mathrm{Au}$ lieu d'être entièrement polarisé par l'objet sacral, but de tout pèlerinage, il est fasciné par son encadrement matériel au point d'être saisi d'envie à la pensée qu'il n'avait rien de comparable dans sa maison : « vœu sacrilège » (659) dont il doit demander pardon à saint Thomas avant de quitter son temple. J'observe en plus que le temps du retour à la vie quotidienne ne manifeste pas, chez Ogyge, les effets attendus d'une véritable transformation spirituelle que provoque normalement le pèlerinage.

Par une dernière flèche décochée contre notre pseudo-pèlerin, Erasme achève son travail de banalisation d'un sacré dévoyé par les intérêts profanes et quasiment ravalé au rang de simple objet de curiosité humaine. Voici la séquence qui me parait l'attester: Ménédème insiste auprès de son ami pour être invité par lui car il brûle, dit-il, d'entendre le reste de l'histoire (fabula). Notre pèlerin lui oppose alors sans vergogne ce marchandage: invitez-moi plutôt demain à déjeuner avec ma femme et «je vous déviderai toute l'histoire jusqu'au soir et jusqu'à plus soif »(865-867)! Quel déficit sacral : l'extraordinaire d'une geste héroïque de rencontre divine s'abîmant en propos anodins de convivialité mondaine !

26 En attendant qu'une hypothétique envie de pérégriner vienne «le chatouiller » (871), Ménédème déclare qu'il a bien assez à faire avec ses «stations romaines» (872). Rien à voir, bien sûr, avec le circuit des églises stationales chères aux « roumieux ». Rome n'est plus dans Rome, si j'ose dire, et le pèlerinage ad limina Apostolorum, le plus prestigieux de la Chrétienté, est remplacé par une ascèse domestique du devoir d'état ${ }^{28}$. Pour 
Ménédème, il n'est d'autre déambulation sacrée qu'à l'intérieur de sa maison pour en régler le bon fonctionnement et veiller, en honnête pater familias, à ce que chacun - femme, enfants, valets et servantes - fasse pleinement son devoir. Voilà l'idéal moral de responsabilité personnelle, la réalité concrète d'un ordre laïc stable que «prescrivent les saintes Écritures » $(880)^{29}$, d'ailleurs en consonnance avec l'idéal païen antique car la mention des «stations romaines » renvoie à un adage d'Erasme (929) : « Le romain remporte la victoire en étant immobile». De cette éthique de la bienséance bourgeoise, il n'est dit nulle part que les saints soient responsables : l'individu chrétien en a la charge. Inutile donc le lointain pèlerinage d'Ogyge à Saint-Jacques pour lui recommander sa famille. Les vraies solidarités se vivent à la maison et c'est le quotidien qu'il faut, si l'on veut, "sacraliser». Autrement dit, le surnaturel est ramené à la quotidiennité d'un exister humain sédentaire mais actif en ses gardes (stationes) domestiques.

De ce texte foisonnant, d'autres lectures restent, à l'évidence, possibles : strictement théologiques mâtinées d'apologétique, préférentiellement littéraires et - pourquoi pas psychanalytiques $^{30}$. Je m'en suis tenu à une approche anthropologique du phénomène pèlerin à la Renaissance, selon son double processus : de désacralisation au vif du discours critique d'Erasme, d'élaboration mentale d'un ordre social neuf. Au fil des analyses, il m'a semblé que se dessinaient à la fois le négatif de la société pèlerine telle qu'elle a pu fonctionner aux siècles du Moyen Age, et l'image floue, encore embrumée, d'une autre société que nous appelons moderne: société de l'éthique personnelle glissant imperceptiblement d'un paradigme religieux, la philosophia Christi érasmienne, absolu christocentrique où se ressourcent toutes les conduites de vie, à des modèles plus ou moins déistes constitutifs d'une morale de plus en plus laïcisée avant de devenir laïque : société de l'homogénéité sociale, avec, organisatrice de ses valeurs, la triade neuve: individu, liberté, conscience. Nouvelle trilogie "sacrée ", réputée commune à tous les membres du corps social, celui-ci finissant par abolir l'antique stratification tripartite des orantes, des militantes et des laborantes ${ }^{31}$. Si Luther a mis en cause plus radicalement qu'Érasme le cloisonnement sacral entre les orantes et tous les autres, c'est-à-dire les laïcs appelés au " sacerdoce universel ", l'ironique et insinuante rhétorique érasmienne, moins outrancière, à dose homéopathique, n'en n'a pas moins contribué surtout post mortem à la démolition de l'ordre ancien. Ce n'est certes pas le but poursuivi par Érasme le modéré ! Il n'empêche: conçue comme un moyen de déblaiement critique pour revenir au christianisme des origines " en esprit et vérité », son œuvre - relue et/ou relayée par des cercles socio-culturels ultérieurs, sceptiques et influents - s'inscrit bel et bien dans le lent processus de désacralisation qui affecte la société occidentale dans son ensemble. Procès qu'en son langage baroque, A. Dupront dénotait comme l'effacement de «la religion panique de salut collectif» qu'incarnait puissamment le pèlerinage, au profit de «cohérences d'exister raisonnables» et d'un «ordre du monde sainement anthropomorphe ».

$28 \mathrm{Au}$ ras de l'événementiel, le décrochement - on le devine - ne se produisit pas sans soubresauts ni crises violentes, par exemple, l'année même de la mort du vieux solitaire de Bâle, dans cette Angleterre érasmienne : au plus fort de la sécularisation henricienne qui provoqua la tragique rébellion $\mathrm{du}$ « christianisme sacral traditionnel $»^{32}$, connue sous le nom de Pilgrimage of Grace (1536-1537), l'homme fort du roi, Thomas Cromwell, fit paraître à des fins de propagande une traduction séparée du Colloque d'Érasme, The pilgrimage of Pure Devotion ${ }^{33}$. 


\section{NOTES}

1. Sur la foisonnante complexité du vocable dans les Adages, voir A. GODIN, « Notes sur Érasme et le sacré ", dans C.M. MURPHY, H. GIBAUD, M.A. di CESARE, eds, Miscellanea moreana. Essays for G. Marc'hadour, Moreana, 100, vol.XXVI, Binghanton, New York, 1989, pp.39-53. Une première version de la présente communication a paru dans les études réunies par Claude BLUM, 1536-1986. Dix conférences sur Érasme. Éloge de la Folie-Colloques, Paris-Genève, 1988.

2. Léon HALKIN, Érasme parmi nous, Paris, Fayard. On lui préfèrera, outre l' Érasme de J. HUIZINGUA, préf. de L. FEBVRE (Les Essais LXXII, Paris, Gallimard, 1945), deux anthologies d'écrits érasmiens par J. CHOMARAT, Érasme. CEuvres choisies, Paris, 1911, et par C. BLUM, A. GODIN, J.C. MARGOLIN, D. MENAGER, Érasme. Textes, Paris, R. Laffont, coll. « Bouquins », à paraître en 1992.

3. F. A. ISAMBERT, Le sens du sacré ; fête et religion populaire, Paris, 1982, p. 303.

4. Alphonse DUPRONT, Du sacré : croisades et pèlerinages. Images et langages, Paris, Gallimard, 1987.

5. F. BIERLAIRE, Les colloques d'Érasme..., Paris, 1978.

6. L.-E. HALKIN, F. BIERLAIRE, R. HOVEN eds, Opera omnia Desiderii Erasmi Roterodami, recognita et adnotatione critica instructa notisque illustrata, Ordinis primi tomus tertius (ASD.1-3), Colloquia, Amsterdam, 1972, pp. 470-494, 881 11. Nous renvoyons entre parenthèses, dans le corps de l'article, aux lignes de cette édition. Traductions quelque peu modifiées d'après V. DEVELAY, Érasme, Les colloques, Paris, 1875, pp. 187-233 ; JAL-PRIEL, Colloques d'Érasme, « les introuvables », Paris, 1983, tome II, pp. 9-64 ; J. CHOMARAT, Anth. cit., pp. 705-745.

7. Voyez les censures de la Faculté de théologie de Paris et les justifications d'Érasme, F. BIERLAIRE, op. cit., pp. 212 sq.

8. Il s'agit d'une tournure assez maniérée, "par préposition impropre ». Cf. ERNOUT-THOMAS, Syntaxe latine, 2e édition, Paris, 1957, p. 117.

9. «Praetextu religionis », De utilitate Colloquiorum, ASD cit., p. 747 ; 1.212. Dans cette apologie, la plus longue séquence porte sur le thème du pèlerinage (11. 35-94): il s'agit bien d'un sujet sensible.

10. Sur le "pèlerinage en esprit », voir A. GODIN, L'homéliaire de Jean Vitrier (T.H.R., $\mathrm{n}^{\circ} \mathrm{CXVI}$ ), Genève, 1971, pp. 44-46, 169, n. 143.

11. Hospites (32); commeantium (156), advenas (603): le premier et le troisième terme se lisent dans Héb. 11, 13 et 1 P. 2, 11.

12. HOMERE, Odyssée, 1,$85 ; 6,72$. «Calypso correspondrait à Notre-Dame des Bords-de-mer (à Walsingham)», J. CHOMARAT, anth. cit., p. 705, note 1. 
13. A. DUPRONT, "Réformes et modernité ", dans B. CHEVALIER et R. SAUZET éds, Les réformes, enracinement socio-culturel, Paris, 1985, p. 422.

14. «Les œufs de serpent " désignant le chapelet, il y a là une trace de raillerie caustique sur le Rosaire, l'une des dévotions les plus populaires de la chrétienté.

15. J. VIEILLARD éd. et trad., Guide du pèlerin de Saint-Jacques de Compostelle, $4 \mathrm{e}$ édition, Mâcon, 1948 ; il s'agit d'un texte latin du XII ${ }^{\mathrm{e}}$ siècle.

16. C'est une hypothèse de A. DUPRONT que l'on trouve dans Saint-Jacques de Compostelle, Paris, 1985, p. 239.

17. La remarque d'Érasme est à rapprocher des expériences, alors en cours dans plusieurs villes d'Empire, pour instituer un système laïc d'assistance publique à la faveur de la sécularisation des biens ecclésiastiques. Cf. J.-L. VIVES, De subventione pauperum, De l'assistance des pauvres, Bruges, mars 1526, trad. Casanova et Caby, Bruxelles, 1943.

18. «Glaucoplutus » transcrit en grec Ulrich, prénom de Zwingli : Eulen-reich, littéralement chouette-riche.

19. Voir F. BIERLAIRE, op. cit., p. 230, note 4.

20. A. DUPRONT, art. «Pèlerinages et lieux sacrés » dans Encyclopedia universalis ; Saint-Jacques de Compostelle, pp. 234-235: procession des géants, le 25 juillet à Compostelle; Du sacré, pp. 384 sq et 392 sq.

21. Cf. 1 P. $1,2$.

22. Ep. $4,13$.

23. A. DUPRONT, « Pèlerinages et lieux sacrés »..., op. cit., col. 733.

24. D. Erasmus Rot. Colloquia familiaria repurgata in usum studiosae juventutis, Ed. novissima, Paris, 1674, p. 360.

25. La connotation sexuelle de secreta est attestée dans la littérature patristique, familière à Erasme. Cf. Ambroise, Tertullien cit. par A. BLAISE, Dictionnaire latin-français des auteurs chrétiens, $746, \mathrm{n}^{\circ} 2$.

26. D. Erasmi Rot..., op. cit. p. 361.

27. Complément à l'allusion faite plus haut (note 17) aux ébranlements socio-économiques dûs au luthéranisme. Dans le Banquet religieux (1522), Erasme s'était montré un peu plus audacieux (voir ASD cit., p. 257, 790 sq).

28. Émule d'Ogyge, Grandgousier, faisant référence à l'enseignement du «bon Apostre sainct Paoul ", dissuade des pèlerins des "otieux et inutiles voyages» et exalte le devoir d'état à la manière d'Érasme: F. RABELAIS, Gargantua, éd. par R. Calder et M.A. Screech, Genève, 1970, p. 256, 11, 95-102.

29. Relevé par J. CHOMARAT, anth. cit., p. 744, note 1.

30. Voir A. GODIN, "Une biographie en quête d'auteur: le Compendium vitae Erasmi», dans Colloque érasmien de Liège, Paris, 1987, pp. 197-222.

31. On trouve de plus amples développements sur ce profil d'analyse dans A. DUPRONT, « Réformes et modernité »... op. cit., pp. 427-434.

32. P. CHAUNU, Église, Culture et Société, Essais sur Réforme et Contre-Réforme (1517-1620), Paris, 1981, p. 255.

33. H. de VOCHT ed., The Earliest English Translations on Erasmus'Colloquia, 1536-1566, Louvain, 1928.

34. Ep. $\mathrm{n}^{\circ} 843,7$ mai 1518 à Martin Lypse : « Fateor de rebus sacris circunspecte loquendum, anxie aut morose non puto». P.S. ALLEN, Opus Epistolarum Des. Erasmi Roterodami, tome III, Oxford, 1913, p. 327, 11, 584-585. 


\section{AUTEUR}

\section{ANDRÉ GODIN}

André GODIN est directeur de recherche au CNRS et directeur du Centre d'Anthropologie Religieuse Européenne à l'EHESS. 